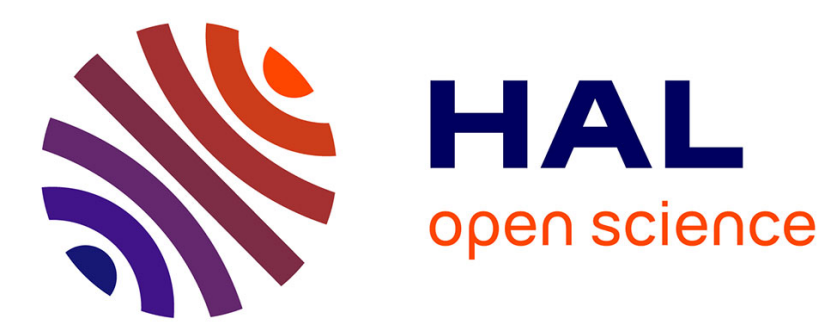

\title{
PROBLÈMES INVERSES ET DÉCONVOLUTION
}

\author{
G. Demoment, J. Idier
}

\section{To cite this version:}

G. Demoment, J. Idier. PROBLÈMES INVERSES ET DÉCONVOLUTION. Journal de Physique IV Proceedings, 1992, 02 (C1), pp.C1-929-C1-936. 10.1051/jp4:19921203 . jpa-00251169

\section{HAL Id: jpa-00251169 https://hal.science/jpa-00251169}

Submitted on 1 Jan 1992

HAL is a multi-disciplinary open access archive for the deposit and dissemination of scientific research documents, whether they are published or not. The documents may come from teaching and research institutions in France or abroad, or from public or private research centers.
L'archive ouverte pluridisciplinaire HAL, est destinée au dépôt et à la diffusion de documents scientifiques de niveau recherche, publiés ou non, émanant des établissements d'enseignement et de recherche français ou étrangers, des laboratoires publics ou privés. 


\title{
PROBLÈMES INVERSES ET DECONVOLUTION
}

\author{
G. DEMOMENT et J. IDIER \\ Laboratoire des Signaux et Systèmes, CNRS/ESE/UPS, Plateau du Moulon, F-91192 Gif-sur-Yvette \\ cedex, France
}

\begin{abstract}
Developments in the theory of image reconstruction and restoration in the past ten or twenty years are outlined. Particular attention is paid to Bayesian inversion methods and to the potential they offer for describing local properties in images.
\end{abstract}

\section{Imagerie acoustique d'un milieu inhomogène}

L'imagerie acoustique active d'un milieu inhomogène consiste à dresser la carte de la répartition spatiale d'une propriété physique de ce milieu, telle que sa célérité ou son impédance, à partir des résultats de son interaction avec une onde acoustique incidente, mesurés en général à la surface de séparation du milieu étudié avec le milieu extérieur. Les techniques correspondantes sont utilisées dans plusieurs domaines tels que la géophysique, le contrôle non destructif et le génie biomédical, et elles sont très variées : échographie ou sismique réflexion, tomographie en réflexion ou en transmission, focalisation synthétique, holographie, migration [1]. La résolution d'un problème d'imagerie peut être fractionnée en trois étapes :

- un problème direct qui consiste, connaissant le milieu et l'onde incidente, à décrire les signaux mesurables dans un formalisme mathématique qui soit à la fois suffisamment précis pour décrire correctement les phénomènes, et assez simple cependant pour se prêter à un traitement numérique ultérieur;

- un problème d'instrumentation qui consiste à générer une onde acoustique incidente adaptée au problème et à mesurer les résultats de son interaction avec le milieu étudié;

- un problème inverse enfin qui consiste, muni du modèle obtenu dans l'étude du problème direct et des données fournies par l'instrumentation, à calculer une image acceptable du milieu.

L'obtention d'une bonne image nécessite évidemment que ces trois sous-problèmes soient étudiés de manière coordonnée. Cet article est consacré aux méthodes de résolution du problème inverse, et plus particulièrement aux méthodes de déconvolution bayésiennes. II a pour but de montrer quelles sont les évolutions majeures qui ont eu lieu ces dernières années dans ce cadre bayésien, et de donner un aperçu des avantages qu'il apporte. Pour illustrer ceci, nous avons choisi un exemple synthétique emprunté à l'échographie en mode $\mathrm{B}$ ou à la sismique réflexion. Il correspond à des méthodes d'imagerie effectivement utilisées en contrôle non destructif, en génie biomédical ou en géophysique. II paraî́tra rudimentaire à beaucoup d'acousticiens, mais nous l'avons cependant retenu car le modèle direct est assez simple pour se prêter à des calculs relativement complets, et illustrer ainsi les possibilités qu'offre l'approche bayésienne pour introduire l'information a priori indispensable à l'obtention d'une image acceptable. C'est en ce sens qu'il présente un caractère générique.

Cet exemple est illustré à la figure suivante. On s'intéresse à un milieu stratifié dont les couches sont supposées homogènes. La figure la représente une section de réflectivité bi-dimensionnelle sur laquelle apparaissent les interfaces entre ces couches. Il s'agit donc de l'objet idéal. L'amplitude des réflecteurs, qui doit être physiquement inférieure à l'unité, ne dépasse pas 0,3 dans cet exemple. Les données sont constituées des traces sismiques de la figure 1c qui fournissent une image dégradée de la section de réflectivité. Ces traces sismiques ont été synthétisées en faisant l'hypothèse que le problème direct pouvait être décrit par une convolution de la réflectivité, dans la direction de propagation, par l'ondelette de la figure 1b, et par l'addition d'un bruit blanc gaussien. Le problème d'imagerie de cet exemple est donc un problème de restauration d'image. Nous supposerons pour simplifier l'exposé que l'ondelette est connue, 
ce qui n'est pas le cas en réalité, mais elle pourrait être calculée sans changer de cadre pour l'inversion, au prix cependant d'une plus grande complexité des calculs.

(a)

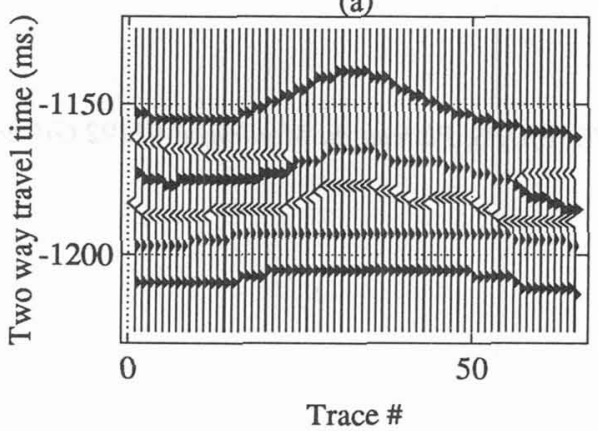

(c)

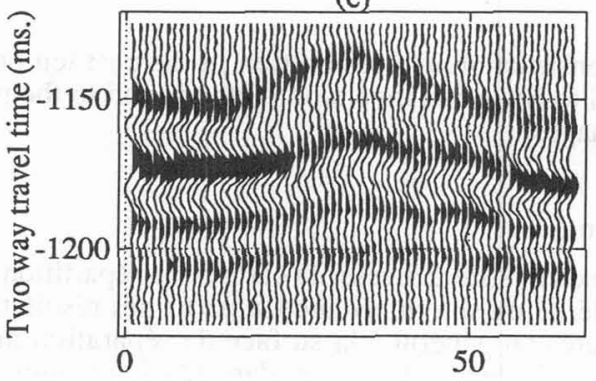

(b)

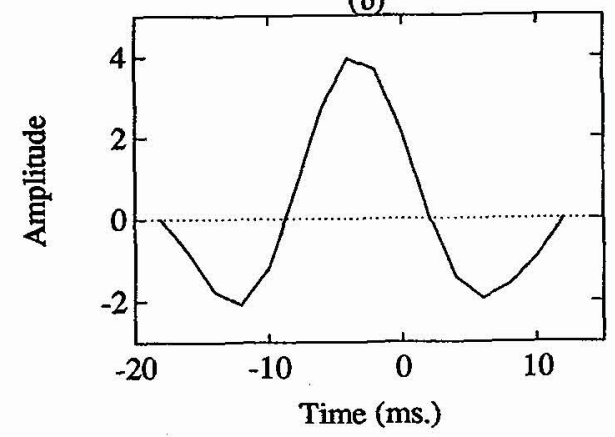

Figure 1- Exemple synthetique : (a) Section de réflectivité recherchée, (b) Ondelette, (c) Trace sismique observée. Le rapport signal-à-bruit est de $10 \mathrm{~dB}$.

Trace \#

\section{Caractère mal-posé du problème de restauration d'image}

Une image est généralement définie comme une fonction à valeurs réelles ou complexes de deux variables appartenant à une région donnée de l'espace. Bien que ce support soit souvent continu, il est habituellement échantillonné sur une grille rectangulaire. Ceci définit un ensemble de pixels, et l'image est représentée par le vecteur $\mathbf{x}$ des $N$ valeurs des intensités des pixels. Dans un problème de restauration d'image, l'objet original ne peut pas être observé directement et doit être restauré à partir des données observées pour éliminer les défauts du mécanisme d'observation.

En restauration d'image, la cause de la dégradation est une distorsion qui peut être décrite par

$$
\mathbf{y}=A(\mathbf{x}) \bullet \mathbf{b}
$$

où $A($.) représente la partie déterministe du mécanisme de dégradation, $\mathbf{b}$ désigne sa composante aléatoire, ou bruit, et le symbole - représente une interaction pixel-à-pixel. Le mécanisme de distorsion implique souvent la convolution de $\mathbf{x}$ par une réponse impulsionnelle et l'addition d'un bruit blanc gaussien indépendant, de moyenne nulle et de variance $\sigma_{i}^{2}$, ce qui conduit au modèle - linéaire - plus simple

Parfois, cependant, la distorsion fait intervenir une transformation non linéaire sur les pixels et un bruit multiplicatif. Le problème de la restauration de $\mathbf{x}$ est alors bien plus difficile [2], même si des méthodes existent formellement dans ce cas. Dans le modèle (2.2), la matrice A et les propriétés statistiques du bruit sont implicitement supposées connues, mais cette hypothèse est rarement satisfaite dans la réalité, et ces quantités doivent être aussi déterminées [2,10,14-15]. C'est justement une situation fréquente en imagerie acoustique. Dans cet article, nous insisterons surtout sur le cas d'une distorsion linéaire connue et d'un bruit gaussien additif. Bien que ce soit quelque peu restrictif, le problème correspondant est générique dans la mesure où sa résolution est à la base de beaucoup d'autres [2-4].

Le problème est d'obtenir une estimée $\hat{\mathbf{x}}$ du vrai objet $\mathbf{x}$ à partir du vecteur des données $\mathbf{y}$. Sous nos hypothèses, la solution des moindres carrés ou de norme minimale émerge immédiatement, qui s'écrit

$$
\hat{\mathbf{x}}_{0}=\left(\mathbf{A}^{t} \mathbf{A}\right)^{-1} \mathbf{A}^{t} \mathbf{y} \text { ou } \hat{\mathbf{x}}_{0}=\mathbf{A}^{\dagger} \mathbf{y} \text {, }
$$

suivant que la matrice normale $\mathbf{A}^{t} \mathbf{A}$ est régulière ou qu'elle n'admet qu'une inverse généralisée. Cela semble un choix raisonnable, d'un point de vue statistique du moins, puisque $\hat{\mathbf{x}}_{0}$ est sous nos hypothèses une solution sans biais et à variance minimale. Mais cette solution est en général inacceptable car A est malconditionnée : l'amplification du bruit qui en résulte dépasse alors tout niveau acceptable. 


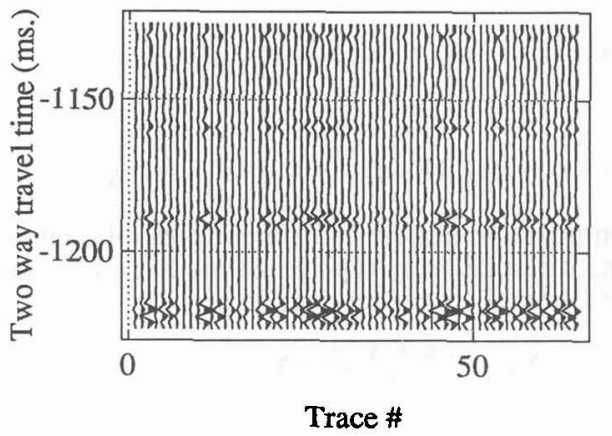

\author{
Figure 2 - Restauration de l'image 1.c. \\ Solution des moindres carrés de norme \\ minimale (inverse généralisée).
}

C'est ce qu'illustre la figure 2 où l'on a tracé la solution inverse généralisée de l'exemple décrit plus haut. La présentation retenue qui est celle des géophysiciens est trompeuse ici à cause de la mise à l'échelle automatique des traces. Il est donc utile de préciser que l'amplitude estimée des réflecteurs atteint plusieurs dizaines d'unités (de -40 à +40 ), ce qui est complètement irréaliste. On ne retrouve pas non plus d'information qualitative correcte sur la géométrie des interfaces entre couches. La solution des moindres carrés est inacceptable, et ce problème se retrouve dans beaucoup d'autres problèmes inverses que la restauration d'image, dans tous les domaines de la physique appliquée. Un principe général pour le résoudre est celui de la régularisation [5-9]. Il consiste essentiellement à changer la notion même de solution puisqu'il est impossible de remonter à la vraie solution à partir de données imparfaites : en régularisant, on considère l'équation initiale (2.2) comme ne définissant en fait qu'une classe de solutions admissibles $\{\hat{\mathbf{x}}\}$

$$
\{\hat{\mathbf{x}}:\|\mathbf{y}-\mathbf{A x}\| \leq\|\mathbf{b}\|\}
$$

parmi lesquelles on doit rechercher une solution acceptable. Pour y parvenir, l'énoncé du problème doit être complété, ce qui implique qu'une information supplémentaire, ou information a priori, y soit incluse.

\title{
3. Régularisation d'un problème mal-posé
}

De nombreuses méthodes ont été proposées pour résoudre et régulariser les problèmes mal-posés. On peut les classer en deux grandes familles : celles qui opèrent dans des espaces fonctionnels et celles qui procèdent par contrôle d'une dimension. Le cadre adopté dans cet article relève de la première famille de méthodes dont la caractéristique principale est de demander à la solution de réaliser un compromis entre une fidélité aux données mesurées et une fidélité à une information a priori. Ce compromis est mesuré à l'aide d'un unique critère d'optimalité. Cette démarche peut s'interpréter de la manière suivante.

La solution des moindres carrés $\hat{\mathbf{x}}_{0}$ minimise l'énergie de l'erreur entre le modèle $\mathbf{A x}$ et les données $\mathbf{y}$. En ce sens, elle procure la plus grande fidélité aux données. Mais lorsque le bruit d'observation est à large bande, les composantes de l'objet restauré ou reconstruit à des fréquences spatiales élevées ont des amplitudes élevées et résultent principalement du bruit. La solution $\hat{\mathbf{x}}_{0}$, bien que sans biais, est en général rejetée car on s'attend à ce que le vrai objet ait des variations spatiales sensiblement plus douces. Il faut donc introduire un peu d'infidélité aux données pour obtenir une solution moins rugueuse que celle des moindres carrés et plus conforme à l'idée que l'on s'en fait a priori. Un moyen simple et très naturel d'y parvenir consiste à définir deux mesures de distance $J_{1}\left(\mathbf{x}, \hat{\mathbf{x}}_{0}\right)$ et $J_{2}\left(\mathbf{x}, \hat{\mathbf{x}}_{\infty}\right)$ entre $\mathbf{x}$ et deux images extrêmes $\hat{\mathbf{x}}_{0}$ et $\hat{\mathbf{x}}_{\infty} . \hat{\mathbf{x}}_{0}$ est la solution ultra rugueuse des moindres carrés, et $\hat{\mathbf{x}}_{\infty}$ correspond à une solution a priori ultradouce. En pratique, $\hat{\mathbf{x}}_{\infty}$ est soit une image d'intensité uniforme, soit une carte de référence fondée sur quelque connaissance a priori. Une solution régularisée $\hat{\mathbf{x}}(\mu, \mathbf{y})$ est alors simplement définie comme la solution du problème suivant

$$
\hat{\mathbf{x}}(\mu, \mathbf{y})=\arg \min _{\mathbf{x} \in X}\left\{J_{1}\left(\mathbf{x}, \hat{\mathbf{x}}_{0}\right)+\mu J_{2}\left(\mathbf{x}, \hat{\mathbf{x}}_{\infty}\right)\right\} .
$$

Il faut noter que $\hat{\mathbf{x}}$ dépend de $\mathbf{y}$ par l'intermédiaire de $\hat{\mathbf{x}}_{0}$. Le choix des mesures $J_{1}$ et $J_{2}$ est un choix qualitatif qui détermine la manière selon laquelle la régularisation est effectuée. Inversement, le choix de $\mu$, qui est le coefficient de régularisation, est quantitatif et permet d'ajuster le compromis entre les deux sources d'information. Une fidélité parfaite aux données est obtenue avec $\mu=0$, alors qu'une fidélité parfaite à l'a priori est obtenue si $\mu=\infty$.

Malgré la généralité du critère (3.1) et les faibles contraintes mathématiques reposant sur les mesures $J_{1}$ et $J_{2}$, seul un petit nombre de distances est effectivement utilisé en reconstruction ou en restauration d'image. A notre connaissance, elles sont toujours choisies parmi les candidates suivantes.

\section{A. Distances quadratiques (euclidiennes) pondérées. La distance euclidienne entre deux images $\mathbf{x}_{1}$ et $\mathbf{x}_{2}$ est}




$$
J_{Q}\left(\mathbf{x}_{1}, \mathbf{x}_{2}\right)=\sum_{i=1}^{N}\left(x_{1 i}-x_{2 i}\right)^{2}=\left(\mathbf{x}_{1}-\mathbf{x}_{2}\right)^{t}\left(\mathbf{x}_{1}-\mathbf{x}_{2}\right)=\left\|\mathbf{x}_{1}-\mathbf{x}_{2}\right\|^{2}
$$

C'est un cas particulier de distance quadratique pondérée

$$
J_{W Q}\left(\mathbf{x}_{1}, \mathbf{x}_{2}\right)=\sum_{i=1}^{N} \sum_{j=1}^{N} w_{i j}\left(x_{1 i}-x_{2 i}\right)\left(x_{1 j}-x_{2 j}\right)=\left(\mathbf{x}_{1}-\mathbf{x}_{2}\right)^{\mathrm{t}} \mathbf{W}\left(\mathbf{x}_{1}-\mathbf{x}_{2}\right)=\left\|\mathbf{x}_{1}-\mathbf{x}_{2}\right\|_{\mathrm{W}}^{2}
$$

où $\mathbf{W}=\operatorname{diag}\left\{w_{i j}\right\}$ est une matrice symétrique, définie non négative qui est choisie pour traduire certaines caractéristiques souhaitées de la mesure de proximité. Une distance de ce type constitue le choix habituel pour $J_{1}$ dans le cas où le bruit $\mathbf{b}$ est supposé blanc, gaussien, centré et indépendant de $\mathbf{x}$

$$
J_{W Q}\left(\mathbf{x}, \hat{\mathbf{x}}_{0}\right)=\sum_{i=1}^{N} \frac{1}{\sigma_{i}^{2}}\left[y_{i}-(\mathbf{A x})_{i}\right]^{2}=\left(\mathbf{x}-\hat{\mathbf{x}}_{0}\right)^{\mathrm{t}} \mathbf{A}^{\mathrm{t}} \Sigma^{-1} \mathbf{A}\left(\mathbf{x}-\hat{\mathbf{x}}_{0}\right)
$$

avec $\Sigma=\operatorname{diag}\left\{\sigma_{i}^{2}\right\}$ et $\mathbf{W}=\mathbf{A}^{\mathfrak{t}} \Sigma^{-1} \mathbf{A}$. Elle est aussi très souvent utilisée pour $J_{1}$ afin de pénaliser les images $\mathbf{x}$ de grande amplitude, ce qui revient à supposer que $\mathbf{x}$ est également blanc, gaussien et centré.

$B$. Mesures de rugosité. Une manière très simple de mesurer la rugosité d'une image consiste à lui appliquer un opérateur de différentiation approprié, puis à calculer la norme euclidienne de l'image résultante. Comme l'opération de différentiation est linéaire par rapport à l'image originale, la mesure de rugosité résultante est quadratique

$$
\Omega(\mathbf{x})=\left\|\nabla^{k}(\mathbf{x})\right\|^{2}=\left\|\mathbf{D}_{k} \mathbf{x}\right\|^{2}=\mathbf{x}^{\mathrm{t}} \mathbf{D}_{k}^{\mathrm{t}} \mathbf{D}_{k} \mathbf{x} .
$$

L'ordre de l'opérateur des différences $\nabla^{k}($.) vaut habituellement $k=1$ ou 2 [2]. $\Omega(x)$ est minimale lorsque toutes les composantes de $\mathbf{x}$ sont égales. Il est donc clair qu'il s'agit d'un cas particulier de distance quadratique pondérée dans laquelle le second argument $\mathbf{x}_{2}$ est une image uniforme et la matrice de pondération est $\mathbf{W}=\mathbf{D}_{k}^{\mathfrak{t}} \mathbf{D}_{k}$.

C. Distances de Kullback. Dans beaucoup de problèmes de traitement d'image, une caractéristique essentielle à préserver est la positivité des intensités des pixels. Il existe plusieurs manières d'y parvenir, l'une d'entre elles étant de considérer que l'objet positif peut être identifié, après normalisation, à une distribution de probabilité, puis d'utiliser des mesures de distance entre lois de probabilité. La pseudodistance de Kullback est souvent utilisée [10-12]

$$
J_{K}\left(\mathbf{x}_{1}, \mathbf{x}_{2}\right)=\sum_{i=1}^{N} x_{1 i} \log \left(x_{1 i} / x_{2 i}\right)
$$

D. Fonctions d'énergie locale. La traduction quantitative de propriétés locales d'une image - douceur, texture,... - peut être envisagée dans le cadre très général des fonctions d'énergie. Un préalable consiste à définir un système de voisinages $\left\{d_{i}\right\}$ dans lequel $d_{i}$ est la collection des pixels qui sont supposés interagir directement avec le pixel $i$. Supposons, à titre d'exemple élémentaire, que les valeurs $x_{i}$ soient quantifiées. Une caractéristique fréquente de beaucoup d'images est qu'il est très probable que les valeurs des intensités en des sites voisins soient elles-mêmes voisines. Aussi on définit les énergies locales

$$
\begin{array}{rlrl}
V\left(x_{i}, x_{j}\right) & =-1 & x_{i}=x_{j} & j \in d_{i}, \\
& =1 & x_{i} \neq x_{j} \quad j \in d_{i}, \\
& =0 & & \text { sinon. }
\end{array}
$$

Ces énergies de liaison, calculées sur tous les pixels voisins, sont ajoutées pour définir l'énergie de l'image

$$
J(\mathbf{x})=\sum_{i} \sum_{j} V\left(x_{i}, x_{j}\right)=\sum_{k} V_{k}(\mathbf{x}) .
$$

$V_{k}$ est l'énergie associée au kième ensemble de pixels voisins, et $k$ indexe l'ensemble de toutes les paires de sites voisins. L'idée suivie dans cette construction est que $J(\mathbf{x})$ doit être faible pour les images qui vérifient les propriétés que l'on veut utiliser pour définir l'a priori, c'est-à-dire dans cet exemple les images dont les pixels voisins ont tendance à avoir les mêmes valeurs d'intensité. Mais les fonctions d'énergie peuvent aussi traduire d'autres propriétés, telles que l'existence de régions presqu'uniformes séparées par des contours marqués. Pour modéliser ces rupture d'homogénéité, des fonctions d'énergie locale ont été récemment introduites [13-16]. L'image originale $\mathbf{x}$ est alors considérée comme une paire $x=(\mathbf{z}, \mathbf{t})$ associant un vecteur d'intensité des pixels $\mathbf{z}$, qui serait directement observable avec un instrument parfait, à un vecteur $\mathbf{t}$ de variables supplémentaires cachées traduisant des caractéristiques inobservables telles que la présence d'un contour, d'une texture, etc... On définit ensuite une énergie globale de la forme

$$
J_{2}\left(\mathbf{x}, \hat{\mathbf{x}}_{\infty}\right)=J_{G}(\mathbf{x})=J_{z}(\mathbf{z})+J_{t}(\mathbf{t})+J_{z t}(\mathbf{z}, \mathbf{t})
$$

composée de trois termes : un terme d'intensité $J_{z}(\mathbf{z})$, un terme de contour $J_{f}(\mathbf{t})$ et un troisième terme $J_{z t}(\mathbf{z}, \mathbf{t})$ qui décrit les interactions nécessaires entre les contours et les pixels [13-16]. Ces énergies locales 
ont des liens étroits avec la physique statistique et les distributions de Gibbs. Malgré cela, leur construction est assez complexe et encore largement empirique.

Après avoir choisi les distances $J_{1}$ et $J_{2}$ la solution régularisée $\hat{\mathbf{x}}(\mu, \mathbf{y})$ est obtenue, pour une valeur de $\mu$ donnée, en minimisant le critère correspondant. C'est là que se posent en général des questions d'ordre pratique sur la complexité des calculs, sur les complications qui découlent du caractère positif de la solution, et de l'existence éventuelle de minima locaux du critère [9]. L'examen de la littérature montre qu'il n'existe pas de réponse satisfaisante aux trois questions simultanées. Les calculs sont en général les plus faciles quand $J_{1}$ et $J_{2}$ sont toutes deux quadratiques

$$
\hat{\mathbf{x}}=\arg \min \left\{\left\|x-\hat{\mathbf{x}}_{0}\right\|_{\mathrm{W}}^{2}+\mu\|\mathbf{x}\|_{\mathrm{K}}^{2}\right\} \text {. }
$$

Ceci correspond aux méthodes de régularisation classiques de Phillips, Twomey et Tykhonov [5-7], qui ont dominé la littérature sur la restauration d'image pendant les quinze dernières années. La solution de (3.9) est unique, linéaire par rapport aux données, et calculable explicitement

$$
\hat{\mathbf{x}}=\left(\mathbf{A}^{\mathrm{t}} \boldsymbol{\Sigma}^{-1} \mathbf{A}+\mu \mathbf{K}\right)^{-1} \mathbf{A}^{\mathrm{t}} \Sigma^{-1} \mathbf{y} \text {. }
$$

Mais cette solution se révèle souvent inadaptée aux exigences du problème. C'est ce qu'illustre le résultat que fournissent ces méthodes lorsqu'on les applique à notre problème synthétique.

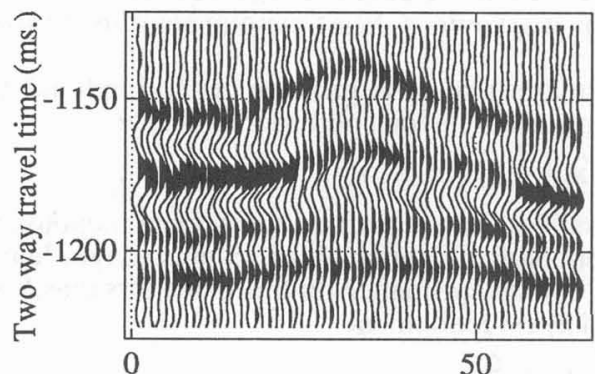

Figure 3 - Restauration de l'image 1.c à l'aide d'une méthode de régularisation classique utilisant des distances quadratiques.

Trace \#

La figure 3 montre la solution régularisée du type (3.10) qui fait simplement l'hypothèse a priori que l'objet recherché est globalement doux. Il y a une amélioration sensible par rapport à la solution des moindres carrés puisque les réflecteurs retrouvent des valeurs physiquement acceptables, mais la résolution de l'image, c'est-à-dire la capacité à discerner des interfaces rapprochées, reste médiocre et on peut même se demander si le résultat apporte sur ce point une amélioration par rapport à l'image brute de la figure 1c. Ce résultat s'explique facilement car la solution dépend linéairement des données. La régularisation effectuée par (3.10) consiste en gros à effectuer une égalisation spectrale dans la bande des fréquences spatiales pour lesquelles le rapport signal-à-bruit est suffisant, et à atténuer considérablement les autres composantes. Avec une ondelette passe-bas comme celle de la figure $1 b$, le spectre utilisable est très réduit et l'amélioration apportée par une telle méthode est nécessairement limitée. On ne peut pas en particulier retrouver une caractéristique essentielle de l'objet de la figure la qui est l'existence d'interfaces nettes séparant des couches homogènes.

Une solution régularisée calculée par (3.10) peut présenter d'autres défauts. Elle n'est, par exemple, pas assurée d'être positive, ce qui est inacceptable dans beaucoup d'applications. Pour y remédier, on peut soit imposer directement la positivité par programmmation non linéaire, soit utiliser une autre distance $J_{2}$, celle de Kullback. Comme la minimisation de $J_{1}\left(\mathbf{x}, \hat{\mathbf{x}}_{0}\right)+\mu J_{2}\left(\mathbf{x}, \hat{\mathbf{x}}_{\infty}\right)$ est équivalente à celle de $J_{2}\left(\mathbf{x}, \hat{\mathbf{x}}_{\infty}\right)+$ (1/ $\mu) J_{1}\left(\mathbf{x}, \hat{\mathbf{x}}_{0}\right)$, ceci conduit aux méthodes dites « à maximum d'entropie » [10]. Malheureusement, on ne peut plus obtenir de solution explicite, et la minimisation du critère doit être faite par itération.

Si l'on veut améliorer la description de l'image en introduisant des contours par exemple, les difficultés de calcul de la solution augmentent encore. Le critère n'a plus de minimum unique, et le minimum global doit être recherché par des techniques assez lourdes.

A ces difficultés de calcul de la solution s'ajoute celle du choix de la valeur à donner au coefficient de régularisation $\mu$ et à d'autres paramètres éventuels caractérisant les distances $J_{1}$ et $J_{2}$. Ce sont les hyperparametres du problème. Il existe plusieurs méthodes pour y parvenir, mais avant de les évoquer, il est utile de présenter une interprétation bayésienne des méthodes de régularisation.

\section{Interprétation bayésienne de la régularisation}

Une part importante des méthodes d'inférence statistique est fondée sur l'emploi d'une information a priori sur les grandeurs à estimer, qui vient s'ajouter à l'information apportée par les données. Il n'est donc pas surprenant, si l'on se réfère à la nature profonde du principe de la régularisation exposé plus haut, qu'il existe un lien étroit entre le principe de la régularisation et l'estimation bayésienne $[2,8]$. Dans un contexte 
statistique, l'information a priori sur l'objet $\mathbf{x}$ s'exprime sous la forme d'une densité de probabilité a priori $f(x)$. La règle de Bayes nous permet de la combiner avec l'information contenue dans les données pour obtenir la loi a posteriori

$$
f(\mathbf{x} \mid \mathbf{y})=f(\mathbf{y} \mid \mathbf{x}) f(\mathbf{x}) / f(\mathbf{y}) \text {. }
$$

Dans cette expression, $f(\mathbf{y} \mid \mathbf{x})$ désigne la loi des données conditionnée par la vraie solution $\mathbf{x}$. Elle est complètement déterminée par la connaissance des modèles (2.1) ou (2.2) et de la loi de probabilité du bruit. Le dernier terme assure la normalisation de la loi a posteriori :

$$
f(\mathbf{y})=\int_{0}^{f} f(\mathbf{y} \mid \mathbf{x}) f(\mathbf{x}) .
$$

Dans un sens strictement bayésien, (4.1) est la solution de l'inversion puisqu'elle résume toutes les informations sur $\mathbf{x}$. Mais la manipulation de densités de probabilité n'est pas très commode et souvent même impraticable, c'est pourquoi une décision doit être prise quant à la valeur à attribuer à chaque pixel. Un choix très fréquent consiste à attribuer à $\mathbf{x}$ la valeur qui maximise la distribution a posteriori $[2,8]$

$$
\hat{\mathbf{x}}=\arg \max f(\mathbf{x} \mid \mathbf{y}) \text {. }
$$

Mais ce n'est qu'une des solutions possible. Cette estimation MAP correspond à la minimisation d'un coût moyen pour une fonction de coût tout-ou-rien. D'autres fonctions de coût ont soulevé récemment l'intérêt dans le cadre de la modélisation des images par champs markoviens. Elles conduisent à maximiser des probabilités marginales [13].

Dans le cas qui nous intéresse ici, il est clair que régulariser selon le principe général indiqué dans le paragraphe précédent est équivalent à choisir l'image qui maximise la loi a posteriori suivante

$$
f(\mathbf{x} \mid \mathbf{y}) \propto \exp \left\{-\frac{1}{2}\left[J_{1}\left(\mathbf{x}, \hat{\mathbf{x}}_{0}\right)+\mu J_{2}\left(\mathbf{x}, \hat{\mathbf{x}}_{\infty}\right)\right]\right\} .
$$

La loi de probabilité précédente n'est qu'un des choix possibles. Toute fonction strictement monotone autre que l'exponentielle aurait convenu. Mais ce choix convient particulièrement bien ici puisqu'avec le modèle linéaire (2.2), sous les hypothèses usuelles de normalité et d'indépendance du bruit, dès lors que $J_{1}=J_{W Q}$ a été choisi avec $\mathbf{W}=\mathbf{A}^{\mathfrak{t} \Sigma^{-1}} \mathbf{A}$, la loi conditionnelle $f(\mathbf{y} \mid \mathbf{x})$ est réellement

$$
f(\mathbf{y} \mid \mathbf{x}) \propto \exp \left\{-\frac{1}{2} J_{1}\left(\mathbf{x}, \hat{\mathbf{x}}_{0}\right)\right\}
$$

Pour que l'analogie soit complète, la loi a priori doit aussi prendre la forme suivante

$$
f(\mathbf{x}) \propto \exp \left\{-\frac{\mu}{2} J_{2}\left(\mathbf{x}, \hat{\mathbf{x}}_{\infty}\right)\right\}
$$

A ce stade, ce n'est qu'une interprétation bayésienne des méthodes de régularisation, et le fait de savoir si cela justifie ou non ces méthodes ne sera pas discuté ici. Indiquons seulement que l'approche bayésienne est en fait le cadre dans lequel les fonctions d'énergie locale ont été introduites en restauration d'image [13$16]$ : par (4.6), ces fonctions définissent $\mathbf{x}$ comme un champ aléatoire markovien.

D'autre part, l'interprétation bayésienne permet d'étendre de manière appréciable la gamme des méthodes de détermination des hyperparamètres [8-9]. Toutes les méthodes décrites ci-dessus nécessitent en effet de connaître la valeur du coefficient de régularisation $\mu$, et plus généralement, de tous les hyperparamètres définissant les mesures de distance $J_{1}$ et $J_{2}$ : les variances du bruit, les paramètres de corrélation de l'objet, et les paramètres des fonctions d'énergie locale. Soit $\theta$ le vecteur de tous ces hyperparamètres. La détermination de $\theta$ est l'étape la plus délicate des méthodes de restauration et de reconstruction d'image. Bien que ce problème ne soit pas encore complètement résolu, la théorie de l'estimation fournit des outils cohérents pour l'aborder.

Citons les méthodes de validation croisée, en particulier pour estimer le paramètre de régularisation $\mu$ dans le cas de distances $J$ quadratiques [17]. Bien que cette méthode présente de bonnes propriétés asymptotiques et soit utilisé couramment dans des problèmes monodimensionnels, il semble avoir été rarement employé en traitement d'image.

Une autre classe de méthodes découle de la définition de la vraisemblance généralisée

que l'on peut maximiser par rapport à $\mathbf{x}$ et à $\theta$

$$
f(\mathbf{x}, \mathbf{y} \mid \boldsymbol{\theta})=f(\mathbf{y} \mid \mathbf{x}, \boldsymbol{\theta}) f(\mathbf{x} \mid \boldsymbol{\theta})
$$

$$
(\hat{\mathbf{x}}, \hat{\theta})=\arg \max _{\mathbf{x} \in X, \theta \in \Theta} f(\mathbf{x}, \mathbf{y} \mid \theta) .
$$

De meilleures propriétés théoriques sont obtenues en maximisant la vraisemblance marginale qui est obtenu en intégrant l'objet hors du problème

$$
f(\mathbf{y} \mid \boldsymbol{\theta})=\int f(\mathbf{y} \mid \mathbf{x}, \boldsymbol{\theta}) f(\mathbf{x} \mid \boldsymbol{\theta}) \mathrm{d} \mathbf{x} .
$$

Mais l'intégrale correspondante est rarement calculable, et on doit s'accommoder d'une technique itérative de maximisation d'espérance conditionnelle souvent coûteuse (algorithme EM [18]). 
Dans le cas des modèles markoviens, il est souvent inconcevable de manipuler une vraisemblance exacte. On peut alors utiliser une pseudo-vraisemblance, construite à partir des lois conditionnelles locales qui sont facilement calculables [14], et dont la maximisation peut alors être faite avec un algorithme EM :

$$
q(\mathbf{y} \mid \theta)=f(\mathbf{y} \mid \mathbf{x}, \theta) q(\mathbf{x} \mid \theta) \text { avec } q(\mathbf{x} \mid \theta)=\prod_{i} f\left(x_{i} \mid x_{j}, \theta ; j \in d_{i}\right) .
$$

Finalement on pourrait aussi, dans un contexte complètement bayésien, attribuer une loi a priori aux hyperparamètres eux-mêmes et les estimer comme l'objet $\mathbf{x}$ par maximum a posteriori. Cette solution à été récemment proposée pour les méthodes à maximum d'entropie, mais il est encore trop tốt pour évaluer leur intérêt dans les problèmes de restauration et de reconstruction d'image.

\section{Imagerie acoustique à l'aide de modèles locaux}

Le premier intérêt de l'approche bayésienne est qu'elle permet d'élargir le choix de l'a priori au travers de la loi $f(x)$. Dans le cas des méthodes de régularisation du type Phillips, Twomey et Tykhonov, la distance $J_{2}$ est une forme quadratique, définie non négative, et (4.6) indique que cela revient à modéliser l'objet comme un champ aléatoire gaussien homogène de moyenne $\hat{\mathbf{x}}_{\infty}$ et de matrice de covariance $\mathbf{W}^{-1}$. Cette matrice peut être choisie de la forme $\left(\mathbf{D}^{\mathrm{t}} \mathbf{D}\right)^{-1}$ si la distance $J_{2}^{\infty}$ est une mesure de rugosité du type (3.4). Plus généralement, elle peut être toute matrice définie non négative, mais sa structure doit être définie à l'avance. Si la matrice est elle-même paramétrée, ses hyperparamètres doivent être déterminés de la même manière que le coefficient de régularisation $\mu$ qui s'interprète dans ce cas comme l'inverse d'un rapport signal-à-bruit.

Dans le cas de l'imagerie acoustique d'un milieu stratifié tel que celui qui est décrit dans notre exemple, un modèle gaussien homogène ne convient manifestement pas puisqu'il ne prend pas en compte l'information essentielle de la présence de contours nets séparant des couches homogènes. Un premier moyen d'y parvenir consiste à utiliser un modèle bernoulli-gaussien au lieu d'un modèle gaussien [19]. L'idée sous-jacente est simple. Une trace sismique verticale idéale est constituée de quelques impulsions isolées séparées par des intervalles de valeurs quasi nulles. Pour traduire ceci, on introduit, en plus des variables aléatoires $\mathbf{x}$ représentant l'amplitude des réflecteurs, des variables aléatoires binaires $\mathbf{q}$ à valeur 0 ou 1 représentant les occurrences de ces impulsions. Ce sont en fait les variables cachées d'un modẻle local monodimensionnel, dont la probabilité $\lambda$ constitue un hyperparamètre supplémentaire du problème.

Une conséquence immédiate de l'emploi d'un modèle bernoulli-gaussien au lieu d'un modèle gaussien est que la complexité des calculs augmente sensiblement. La maximisation exacte de la probabilité a posteriori de la solution devient un problème combinatoire qui nécessite de résoudre $2^{\mathrm{N}}$ problèmes élémentaires comparable chacun à celui d'une restauration gaussienne. En pratique, on utilise des techniques de maximisation approchée [19]. La figure 4 montre le résultat que l'on obtient avec un tel modèle sur l'exemple de la figure 1. L'amélioration par rapport au cas gaussien est sensible car l'objet restauré présente cette fois la caractéristique souhaitée de ne contenir qu'un petit nombre de réflecteurs. L'allure générale des interfaces est bien restituée, même si l'on observe un certain nombre d'erreurs dans la localisation des réflecteurs dues à des erreurs de détection ou à des fausses alarmes.

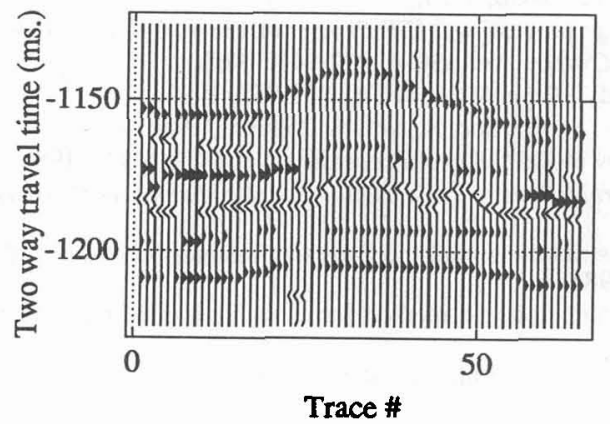

Figure 4-Restauration de l'image 1.c à l'aide d'une méthode de régularisation bayésienne utilisant un modele local 1-D du type bernoulli-gaussien.

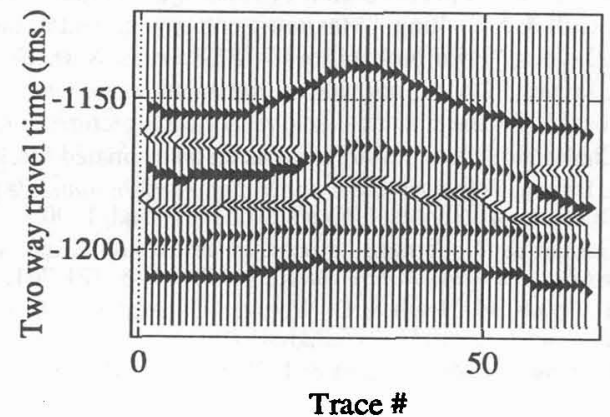

Figure 5-Restauration de l'image 1.c à l'aide d'une méthode de régularisation bayesienne utilisant un modele local 2-D du type markov-bemoulli-gaussien.

Dans la méthode de restauration précédente, les modèles utilisés, aussi bien pour décrire l'objet que la dégradation, sont monodimensionnels et chacune des traces sismiques est en fait traitée indépendamment de ses voisines. Il est clair que cela ne permet pas de traduire une autre caractéristique importante de l'objet qui 
est sa structure transversale. Il s'agit, rappelons-le, de la coupe verticale d'un milieu stratifié dont les couches sont régulièrement disposées les unes au dessus des autres. Les interfaces sont donc continues et orientées préférentiellement vers l'horizontale. Il faut donc, pour traduire ce caractère de l'objet, introduire une corrélation spatiale que ne possède pas le modèle bernoulli-gaussien. C'est ce que permet de faire le modèle markov-bernoulli-gaussien [20-21]. Un second niveau de variables binaires cachées, les variables de transition $\mathbf{t}$, sont introduites pour relier entre eux les réflecteurs de deux traces voisines appartenant à la même interface. Le modèle se complique quelque peu puisqu'il est nécessaire de faire appel à cinq hyperparamètres supplémentaires pour donner une souplesse suffisante au modèle et autoriser les interfaces à se courber, à se dédoubler, etc... La figure 5 montre le résultat d'une restauration obtenue avec un tel modèle local bidimensionnel. La comparaison avec la figure 1a indique que cette fois, toutes caractéristiques essentielles de l'objet ont bien été retrouvées.

\section{Conclusions}

Le traitement du signal et de l'image est une discipline d'ingénieur qui a connu des développements considérables depuis quelques années, sous la poussée du développement propre des disciplines plus théoriques dont il s'inspire, comme la statistique, mais surtout à cause de l'évolution étonnante de la microinformatique qui lui fournit de moyens de traitement de plus en plus puissants. Il devient donc possible d'effectuer maintenant des traitements d'une très grande complexité, inenvisageables il y a encore quelques années. Les conséquences de ces changements sont très importantes pour l'imagerie en général puisqu'il devient possible d'utiliser des traitements statistiques qui ont fait la preuve de leur efficacité depuis longtemps dans d'autre domaines moins complexes, avec des modèles de plus en plus sophistiqués. Il est clair en particulier que les méthodes d'inversion bayésiennes sont appelées à jouer un rôle de plus en plus important dans ce domaine, et nous espérons que cet article aura contribué à attirer l'attention des acousticiens sur les possibilités de plus en plus nombreuses qu'elles leur apportent.

\section{Bibliographie}

[1] F. Champagnat, Méthodes d'imagerie acoustique, LSS Int. Rep. N GPI-91/01, 1991.

[2] H.C. Andrews \& B.R. Hunt, Digital image restoration, Englewood Cliffs: Prentice-Hall, 1977.

[3] G.T. Herman, H.K. Tuy, H. Langenberg \& P.C. Sabatier, Basic methods of tomography and inverse problems, Adams Hilger Publ., 1987.

[4] A.C. Kak \& M. Slaney, Principles of computerized tomographic imaging, New-York: IEEE Press, 1988.

[5] M.Z. Nashed, "Operator-theoretic and computational approaches to ill-posed problems with applications to antenna theory," IEEE Trans. Antennas Propagat, vol.AP-29, 220-231, 1981.

[6] J.L.C. Sanz \& T.S. Huang, "Unified Hilbert space approach to iterative least-squares linear signal restoration", $J . O p t$. Soc. Am., vol.73, 1455-1465, 1983.

[7] A. Tikhonov \& V. Arsenin, Solutions of ill-posed problems, Washington DC: Winston \& Sons, 1977.

[8] D.M. Titterington, “Common structure of smoothing techniques in statistics", Intern. Statist. Rev., vol.53, 141-170, 1985.

[9] G. Demoment, "Image reconstruction and restoration : overview of common estimation structures and problems", IEEE Trans. Acoust., Speech, Signal Processing, vol.ASSP-37, 2024-2036, 1989.

[10] S.F. Gull \& J. Skilling, "Maximum entropy method in image processing," IEE Proc., vol.131-F, 646-659, 1984.

[11] E.T. Jaynes, "Prior probabilities," IEEE Trans. Syst., Sc., Cybern., vol.SSC-4, 227-241, 1968.

[12] E.T. Jaynes, “On the rationale of maximum-entropy methods," Proc. IEEE, vol.70, 939-952, 1982.

[13] J. Besag, "On the statistical analysis of dirty pictures", J. R. Statist. Soc. B., vol.48, 259-302, 1986.

[14] B. Chalmond, "Image restoration using an estimated Markov model", Signal Processing, vol.15, 115-129, 1988.

[15] J.M. Dinten, Tomographie à partir d'un nombre limité de projections : régularisation par des champs markoviens, Thèse de Doctorat en sciences, Université de Paris-Sud, 1990.

[16] S. Geman \& D. Geman, "Stochastic relaxation, Gibbs distributions, and the Bayesian restoration of images", IEEE Trans.Pattern Anal. Mach. Intell., vol.PAMI-6, 721-741, 1984.

[17] G.H. Golub, M. Heath \& G. Wahba, "Generalized cross-validation as a method for choosing a good ridge parameter", Technometrics, vol.21, 215-223, 1979.

[18] A.P. Dempster, N.M. Laird \& D.B. Rubin, "Maximum likelihood from incomplete data via the EM algorithm", $J . R$. Statist. Soc. B., vol.39, 1-38, 1977.

[19] Y. Goussard \& G. Demoment, "Recursive deconvolution of Bernoulli-Gaussian processes using a MA representation", IEEE Trans. Geoscience \& tremote Sensing, vol.27, 384-394, 1989.

[20] J. Idier \& Y. Goussard, "Markovian modeling for Bayesian multi-chanel deconvolution", Proc. IEEE Intern. Conf. Acoust., Speech, Signal Processing, Albuquerque, Apr.1990, 2001-2004.

[21] J. Idier \& Y. Goussard, "Markovian modeling for Bayesian restoration of 2-D layered structures", LSS Int. Rep. N GPI89/06, 1989. 